\title{
The prognostic utility of haptoglobin genotypes in squamous cell carcinoma of the head and neck
}

\author{
Ching-Chih Lee ${ }^{1,2,3}$, Moon-Sing Lee ${ }^{3,4}$, \\ Hsu-Chueh $\mathrm{Ho}^{2,3}$, Shih-Kai Hung ${ }^{3,4}$, Ya-Ting \\ Tung $^{5}$, Pesus Chou ${ }^{1}$ and $\mathrm{Yu}-\mathrm{Chieh} \mathrm{Su}^{3,5, *}$ \\ ${ }^{1}$ Community Medicine Research Center and Institute \\ of Public Health, National Yang-Ming University, \\ Taipei, Taiwan \\ ${ }^{2}$ Department of Otolaryngology, Buddhist Dalin Tzu \\ Chi General Hospital, Chiayi, Taiwan \\ ${ }^{3}$ School of Medicine, Tzu Chi University, Hualian, \\ Taiwan \\ ${ }^{4}$ Department of Radiation Oncology, Buddhist Dalin \\ Tzu Chi General Hospital, Chiayi, Taiwan \\ ${ }^{5}$ Division of Hematology-Oncology, Department of \\ Internal Medicine, Buddhist Dalin Tzu Chi General \\ Hospital, Chiayi, Taiwan
}

\begin{abstract}
Background: The aim of this study was to determine whether haptoglobin ( $\mathrm{Hp}$ ) genotypes are associated with prognosis in patients with squamous cell carcinoma of the head and neck (HNSCC).

Methods: We studied patients with HNSCC without distant metastasis at diagnosis. The Hp genotype of each patient was determined and the prognostic significance of the Hp genotype was further analyzed. Pearson's $\chi^{2}$-test or Fisher's exact test were used to analyze correlations between Hp genotype and clinical characteristics of HNSCC. Eighty patients with newly diagnosed HNSCC who were treated with curative modality were enrolled in this study. KaplanMeier plots and log-rank test were used to compare locoregional recurrence-free survival, distant-metastasis-free survival and overall survival of patients according to $\mathrm{Hp}$ genotype. Survival analysis was performed using Cox proportional hazard models.

Results: Eighty patients with newly diagnosed HNSCC were enrolled in this study. There was no significant difference in the distribution of $\mathrm{Hp}$ genotypes in HNSCC patients and healthy individuals $(p=0.959)$. Matched-pair analysis showed that locoregional recurrence-free survival was poor $(p=0.02)$ for HNSCC patients with Hp 2-2 or 2-0. There was no significant difference in distant metastasis-free survival and overall survival $(p=0.422$ and 0.509 , respectively). Multivariate analysis showed that $\mathrm{Hp} \mathrm{2-2}$ or 2-0 was associated with an increased risk of locoregional

*Corresponding author: Yu-Chieh Su, Division of Hematology-Oncology, Department of Internal Medicine, Buddhist Tzu Chi Dalin General Hospital, No. 2 Min-Sheng Road, Dalin, Chiayi 622, Taiwan

Phone: +886 52854000 Ext. 5231, Fax: +886 52648006 ,

E-mail: hematcd@hotmail.com

Received May 11, 2009; accepted July 7, 2009;

previously published online September 11, 2009
\end{abstract}

recurrence [Hazard ratio (HR) 5.9; 95\% confidence interval (Cl), 1.1-6.65; $\mathrm{p}=0.038]$. The risk was still higher in patients with $\mathrm{Hp} \mathrm{2-2}$ or 2-0 after further adjusting for age and treatment modality (HR 7.6; 95\% $\mathrm{Cl}, 1.2-46 ; \mathrm{p}=0.028$ ) in locoregional recurrence-free survival.

Conclusions: The present data show that the $\mathrm{Hp}$ genotype is closely related to recurrence rate in patients with HNSCC. Patients with $\mathrm{Hp} \mathrm{2-2}$ or 2-0 have greater locoregional recurrence and significantly increased HRs in multivariate analysis. The Hp genotype may be a prognostic factor in patients with HNSCC.

Clin Chem Lab Med 2009;47:1277-83.

Keywords: genotype; haptoglobin; head and neck squamous cell carcinoma; survival.

\section{Introduction}

Squamous cell carcinoma of the head and neck (HNSCC) is one of the most common cancers worldwide that occurs predominantly in middle-aged and older men who use tobacco, alcohol, and betel nuts. In Taiwan, the incidence of HNSCC has continued to increase and is currently the fourth most common cause of cancer-related mortality in men (1). HNSCC has a high recurrence rate and poor survival. The prognosis and clinical outcomes of patients with HNSCC depends primarily on the TNM staging system. However, the most popular and widely used TNM system does not always predict prognosis well. Reliable prognostic factors may be used to supplement the present staging system.

Haptoglobin ( $\mathrm{Hp})$, a hepatocyte-derived serum $\alpha_{2}$ glycoprotein, is an acute-phase reactant produced in response to inflammatory cytokines and glucocorticosteroids $(2,3)$. Previous studies have shown that $\mathrm{Hp}$ may play an important role in host defense against infection and neoplasms by decreasing the availability of iron to invading pathogens and tumor cells, and by modulating angiogenesis and other inflammatory processes $(4,5)$. The expression of $\mathrm{Hp}$ is controlled by the polymorphic $\mathrm{Hp}$ locus located on chromosome 16q22. The characteristics of $\mathrm{Hp}$ vary according to the different $\mathrm{Hp}$ genotypes. Hp 1-1 is a much better antioxidant compared with HP 2-2 or HP 2-0, and binds more strongly with free hemoglobin $(3,6,7)$. Angiogenic effects are more frequently observed with $\mathrm{Hp}$ 2-2, while $\mathrm{Hp} \mathrm{2-1}$ has an intermediate characteristic $(8,9)$.

Several studies have investigated the relationship between $\mathrm{Hp}$ concentrations and head and neck cancer $(10,11)$. However, there are no published data on the 
prognostic influence of Hp genotypes in HNSCC. The goal of this study was to investigate the prognostic influence of Hp genotypes in HNSCC.

\section{Materials and methods}

\section{Human subjects}

The study was conducted with the approval of the Human Research Ethics Committees of our institution. Written informed consent was obtained from all patients with HNSCC and healthy individuals between 2005 and 2008. A total of 92 cases were identified and retrieved from the archives. Twelve patients were not eligible for analyses because of the presence of distant metastasis at the time of presentation and loss of follow-up. In this study, healthy subjects, who were asymptomatic and underwent physical examination, chest $\mathrm{X}$-ray, abdominal sonogram for reasons of promoting good health and disease prevention during annual health check-up were recruited as control subjects. All blood samples were collected in EDTA-containing tubes. A total of 80 HNSCC patients for whom complete data sets were available were analyzed. The following data were recorded for each patient: age, gender, tobacco use, alcohol use, betel nut chewing, treatment modality, and outcome. All tumors were staged according to the American Joint Committee on Cancer (AJCC) system, modified in 2002.

All patients initially received curative treatment. On completion of treatment, patients were followed regularly every month during the first year, every 2 months in the second year and every 3 months thereafter. Chest $X$-rays were performed annually, while head and neck magnetic resonance imaging/computed tomography, bone scans and ultrasound of abdomen were performed when clinically indicated.

\section{Hp genotyping based on polymerase chain reaction ( $P C R$ )}

Genomic DNA was extracted from peripheral blood mononuclear cells using a DNA extraction kit (Qiagen, Valencia, CA, USA). PCR methods were used to analyze the $\mathrm{Hp} 1$ and Hp 2 alleles as described previously $(12,13)$. Primers Hp-del$\mathrm{U}$ and Hp-del-L were used for amplification of a 315-base pair (bp) $\mathrm{Hp} \mathrm{O}$ allele-specific sequence. Primers Hp-exon-U and $\mathrm{Hp}$-exon-L were used to amplify a $476-\mathrm{bp} \mathrm{Hp} 1$ or $\mathrm{Hp} 2$ allele-specific sequence $(14,15)$. PCR was carried out in a final volume of $50 \mu \mathrm{L}$ containing $10-20 \mathrm{ng}$ of genomic DNA.

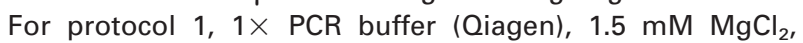
$0.2 \mathrm{mM}$ dNTPs, $0.4 \mu \mathrm{M}$ of each primer, and 2 units of Qiagen tag DNA polymerase were included in the PCR reactions using primers $A$ and $B$. The program for $P C R$ using primers $A$ and $B$ was as follows: an initial incubation at $95^{\circ} \mathrm{C}$ for $5 \mathrm{~min}, 35$ cycles of incubation at $95^{\circ} \mathrm{C}$ for $1 \mathrm{~min}, 66^{\circ} \mathrm{C}$ for $40 \mathrm{~s}$, and $72^{\circ} \mathrm{C}$ for $90 \mathrm{~s}$, with a final extension at $72^{\circ} \mathrm{C}$ for $10 \mathrm{~min}$. For protocol 2, 1× PCR buffer (Qiagen), $1.5 \mathrm{mM}$ $\mathrm{MgCl}_{2}, 0.05 \mathrm{mM}$ dNTPs, $0.2 \mu \mathrm{M}$ of each primer, and 2 units of ABgene tag DNA polymerase were included in the PCR reactions using primers $C$ and $D$. For the PCR with primers $C$ and $D$, the program was as follows: an initial incubation at $95^{\circ} \mathrm{C}$ for $5 \mathrm{~min}, 35$ cycles of incubation at $95^{\circ} \mathrm{C}$ for $1 \mathrm{~min}$, $69^{\circ} \mathrm{C}$ for $1 \mathrm{~min}$, with a final extension at $72^{\circ} \mathrm{C}$ for $10 \mathrm{~min}$. For PCR protocol 3, $1 \times$ PCR buffer (Qiagen), $1.5 \mathrm{mM} \mathrm{MgCl}_{2}$, $0.2 \mathrm{mM}$ dNTPs, $0.2 \mu \mathrm{M}$ of each primer, and 2 units of Qiagen taq DNA polymerase were included in the PCR reactions. The thermal cycler conditions used for amplification were as follows: an initial incubation at $95^{\circ} \mathrm{C}$ for $5 \mathrm{~min}, 35$ cycles of incubation at $95^{\circ} \mathrm{C}$ for $30 \mathrm{~s}, 60^{\circ} \mathrm{C}$ for $40 \mathrm{~s}$, and $72^{\circ} \mathrm{C}$ for $1 \mathrm{~min}$, with a final extension at $72^{\circ} \mathrm{C}$ for $10 \mathrm{~min}$. The resulting PCR products underwent electrophoresis in $1 \%$ agarose gel stained with ethidium bromide. All patient samples were analyzed using protocol 1 with primers $A$ and $B$. If the presence of the 1757-bp product was detected, the samples were later analyzed according to protocol 2 with primers $C$ and $D$. The Hp 0-specific PCR product was detected as a 315-bp band, whereas the product of $\mathrm{Hp}$ exon 1 was detected as a 476-bp band. The sequences of primers were:

A: 5'-GAGGGGAGCTTGCCTTTCCATTG-3'

B: 5'-GAGATTTTTGAGCCCTGGCTGGT-3'

C: 5'-CCTGCCTCGTATTAACTGCACCAT-3'

D: 5'-CCGAGTGCTCCACATAGCCATGT-3'

Hp-del-U: 5'-CTTTATGGCACTGGGGAACAAGCATTTTG-3'

Hp-del-L: 5'-CAGGAAGAGATTTTTAGCCGTGGTCAGCAG-3' Hp-exon-U: 5'-GCAGTGTGAAAATCCTCCAAGATAA-3' Hp-exon-L: 5'-AATTTAGCCCATTTGCCCGTTTCTT-3'.

\section{Analyzing survival rates with matched-pair method}

In order to limit the effect of confounders and to increase statistical efficiency, a matched-pair method was conducted. Previous studies showed that patients with nasopharyngeal carcinoma and breast cancer who had the Hp 1-1/2-1 genotype had a better prognosis $(16,17)$. Due to these observations, we assigned HNSCC patients into two groups: patients with $\mathrm{Hp} 1-1$ or 2-1 and patients with $\mathrm{Hp} \mathrm{2-2}$ or 2-0. Patients with $\mathrm{Hp}$ 1-1 or 2-1 were matched randomly with patients with Hp 2-2 or 2-0 in a 1:1 ratio. Matching variables were gender, site of primary tumor, and the stage of clinical disease.

\section{Statistical analysis}

All data were analyzed using the SPSS (Version 12, SPSS Inc., Chicago, IL, USA) system. The association between different categorical variables was analyzed with Pearson's $\chi^{2}$-test or Fisher's exact test. Hardy-Weinberg equilibrium analyses for $\mathrm{Hp}$ polymorphism were conducted with one degree of freedom in the HNSCC patients and controls. Survival rates were estimated using the Kaplan-Meier method and Kaplan-Meier survival curves were compared using the log-rank test. The Cox proportional regression model was used to evaluate the effect of $\mathrm{Hp}$ genotypes on survival rates after adjusting for matching variables and other potential prognostic factors.

\section{Results}

The following $\mathrm{Hp}$ genotype-specific banding was obtained: Hp 1-1 and Hp 2-2 were characterized by single bands representing the 1757- and 3481-bp products, respectively. Genotype 2-1 was characterized by the presence of both the 1757- and 3481-bp

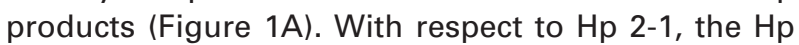
1-specific 1757-bp band was generally more intense than the Hp 2-specific 3481-bp band (Figure 1A, lane 2). Figure $1 B$ shows that patients without the $349-b p$ product had the Hp 1-1 genotype (lane 5). Figure 1C shows the Hp 0 pattern.

Figure 2 shows patient enrollment in this study. A total of 80 patients with HNSCC with complete data were enrolled. Also, 80 healthy individuals of similar age and gender were included as a comparison group for the distribution of Hp genotypes. The final study sample of matched-pair analysis consisted of 46 
A

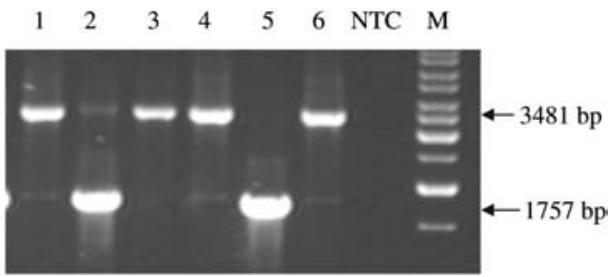

Genotype

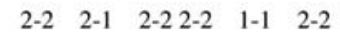

$\mathbf{B}$

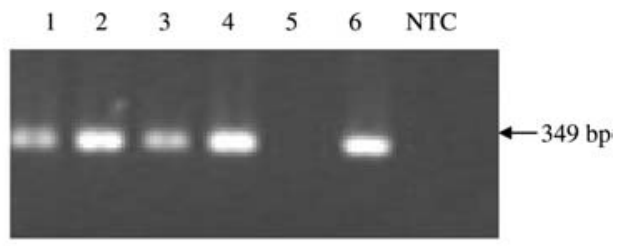

C

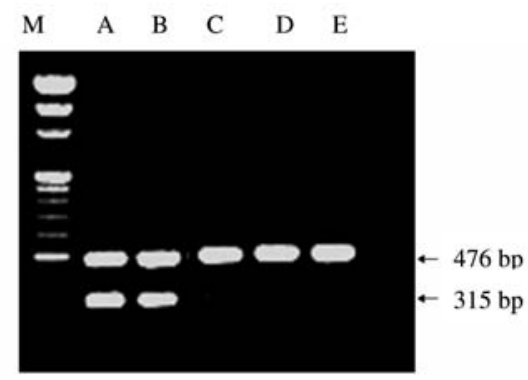

Figure 1 Haptoglobin genotyping. Agarose gels demonstrating genotype determinations using DNA from individuals representing genotype $\mathrm{Hp} \mathrm{1-1,} \mathrm{Hp} \mathrm{2-1} \mathrm{and} \mathrm{Hp}$ 2-2.

(A) Haptoglobin genotyping with primer A and B (protocol 1). (B) Protocol 2 with primer $C$ and $D$ was used to determine the complete genotype when the 1757-bp band was detected. A 349-bp product was generated from the genomic DNA of individuals homozygous or heterozygous for the $\mathrm{Hp} 2$ allele, whereas no product was formed in the presence of the $\mathrm{Hp} 1$ allele (lane 5, DNA from the individuals with the $\mathrm{Hp}$ 1-1 genotype). (C) Detection of haptoglobin gene deletion. Both 315-bp and 476-bp bands were amplified from individuals heterozygous for the $\mathrm{Hp} \mathrm{O}$ allele (lanes $\mathrm{A}$ and $\mathrm{B}$ ).

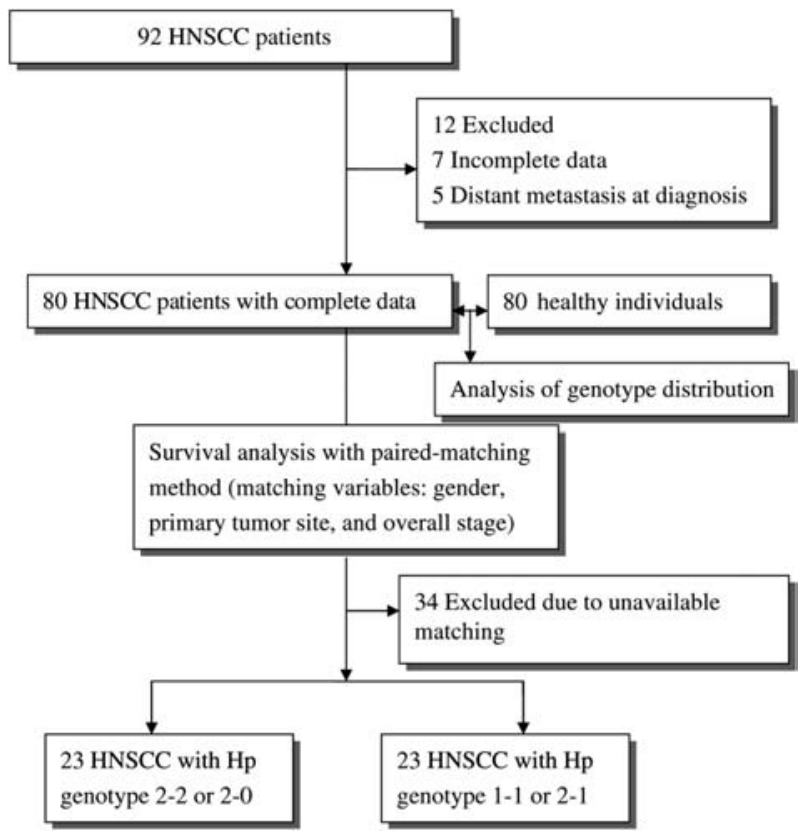

Figure 2 Flow diagram for analysis of genotype distribution and survival analysis with paired-matching method. patients with HNSCC (44 men, 2 women; mean age, 51 years), 23 patients with $\mathrm{Hp} 1-1$ or 2-1 and 23 patients with $\mathrm{Hp} \mathrm{2-1}$ or $\mathrm{Hp} \mathrm{2-0.} \mathrm{In} \mathrm{each} \mathrm{group,} \mathrm{there}$ were 11 patients with oral cancer, four patients with oropharyngeal cancer, and eight patients with hypopharyngeal cancer.

\section{Association between HNSCC and Hp genotypes}

The first part of the study was comprised of 80 HNSCC patients and 80 healthy individuals. Table 1 shows the distribution of $\mathrm{Hp}$ genotypes in healthy individuals and patients with HNSCC. There was no significant difference in the distribution of Hp genotypes between these two groups $(p=0.959)$. The distribution of genotypes in healthy individuals and HNSCC patients were in Hardy-Weinberg equilibrium $(p=0.139$ and 0.113 , respectively).

\section{Association between Hp genotypes and survival of HNSCC patients}

The demographics of the two groups were similar. There were no significant differences between the two populations in terms of gender, tobacco use, alcohol use and betel nut chewing. The patients were evenly matched with respect to tumor stage, tumor classification, and nodal status (Table 2). Although the two groups were not matched with respect to treatment modality, the types of treatment received were similar.

The time of follow-up ranged from 5 to 60 months [mean \pm standard deviation (SD), $21 \pm 11.7$ months; median, 21 months] for the HNSCC patients with $\mathrm{Hp}$ 1-1 or 2-1, and ranged from 4 to 52 months (mean \pm SD, $20 \pm 10.2$ months; median, 19 months) for the HNSCC patients with $\mathrm{Hp} \mathrm{2-2}$ or 2-0. HNSCC

Table $1 \mathrm{Hp}$ genotype distribution in the normal control group and HNSCC patients.

\begin{tabular}{llll}
\hline Variables & $\begin{array}{l}\text { Control group } \\
(\mathrm{n}=80)\end{array}$ & $\begin{array}{l}\text { HNSCC group } \\
(\mathrm{n}=80)\end{array}$ & p-Value \\
\hline $\begin{array}{l}\text { Gender } \\
\text { Male }\end{array}$ & $78(69)$ & $78(69)$ & \\
$\quad$ Female & $2(31)$ & $2(31)$ & 0.652 \\
$\begin{array}{l}\text { Age } \\
\text { Mean } \pm \text { SD }\end{array}$ & $51 \pm 12$ & $52 \pm 11$ & \\
Genotype & & & 0.959 \\
Hp 1-1 & $8(10)$ & $8(10)$ & \\
Hp 2-1 & $35(44)$ & $32(40)$ & \\
Hp 2-2 & $33(41)$ & $35(44)$ & \\
Hp 2-0 & $4(5)$ & $5(6)$ & \\
$\chi^{2 \text { a,b }}$ & 2.179 & 2.5 & \\
Alle frequency & & & \\
Hp 1 & 0.319 & 0.3 & \\
Hp 2 & 0.656 & 0.669 & \\
Hp 0 & 0.025 & 0.031 & \\
\hline
\end{tabular}

Values are given as number (percentage). ${ }^{a} \chi^{2}$-test for deviation from Hardy-Weinberg equilibrium; ${ }^{a} p$-values from goodness-of-fit $\chi^{2}$-test in Hardy-Weinberg equilibrium in the control group was 0.139 , and 0.113 in the HNSCC group. HNSCC, head and neck squamous cell carcinoma; SD, standard deviation; Hp, haptoglobin. 
Table 2 Characteristics of patients: matching variables.

\begin{tabular}{|c|c|c|c|}
\hline & \multicolumn{2}{|l|}{ Genotype } & \multirow[t]{2}{*}{$\mathrm{p}$-Value } \\
\hline & $\begin{array}{l}\text { Hp 1-1, 2-1 } \\
(n=23)^{a}\end{array}$ & $\begin{array}{l}\text { Hp 2-2, 2-0 } \\
(\mathrm{n}=23)^{\mathrm{b}}\end{array}$ & \\
\hline \multicolumn{4}{|l|}{ Gender } \\
\hline Male & $22(96)$ & $22(96)$ & \\
\hline Female & $1(4)$ & $1(4)$ & \\
\hline \multicolumn{4}{|l|}{ Age in years } \\
\hline Mean $\pm S D$ & $52 \pm 12$ & $50 \pm 11$ & 0.739 \\
\hline \multicolumn{4}{|l|}{ Primary site } \\
\hline Oral cavity & 11 & 11 & \\
\hline Oropharynx & 4 & 4 & \\
\hline Hypopharynx & 8 & 8 & \\
\hline \multicolumn{4}{|l|}{ Stage } \\
\hline III & $1(4)$ & $1(4)$ & \\
\hline IV & $22(96)$ & $22(96)$ & \\
\hline T classification & & & 0.562 \\
\hline T2 & $7(30)$ & $4(17)$ & \\
\hline Т3-4 & $16(70)$ & $19(83)$ & \\
\hline $\mathrm{N}$ classification & & & 1 \\
\hline N0-1 & $7(30)$ & $7(30)$ & \\
\hline N2-3 & $16(70)$ & $16(70)$ & \\
\hline Treatment & & & 0.475 \\
\hline $\begin{array}{l}\text { Surgery+adjuvant } \mathrm{R} / \mathrm{T} \\
\text { or adjuvant CCRT }\end{array}$ & $17(74)$ & $19(83)$ & \\
\hline CCRT & $6(26)$ & $4(17)$ & \\
\hline
\end{tabular}

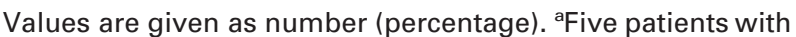

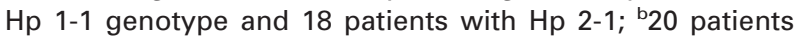
with Hp 2-2 genotype and three patients with $\mathrm{Hp}$ 2-0 genotype. Hp, haptoglobin; SD, standard deviation; $\mathrm{R} / \mathrm{T}$, radiotherapy; CCRT, concurrent chemoradiotherapy.

patients with Hp 1-1 or 2-1 had better locoregional recurrence-free survival rates $(p=0.02$; Figure $3 A)$. Table 3 shows the failure patterns of HNSCC patients with different Hp genotypes. Ten of 23 (44\%) HNSCC patients with $\mathrm{Hp}$ 2-2 or 2-0 had locoregional recurrence, vs. three of $23(13 \%)$ HNSCC patients with $\mathrm{Hp}$ 1-1 or 2-1 $(p=0.022)$. Patients with recurrent tumor received salvage treatment. Seven of 10 HNSCC patients with $\mathrm{Hp}$ 2-2 or 2-0 received salvage surgery with or without adjuvant chemoradiotherapy and four patients were successfully salvaged; three patients received concurrent chemoradiotherapy for their recurrent tumor and one patient was successfully salvaged. Two of three HNSCC patients with Hp 1-1 or 2-1 received surgical intervention and neither of these patients were successfully salvaged; one patient who received chemotherapy was successfully salvaged. There was no significant difference with respect to distant metastasis-free survival and overall survival rates $(p=0.422$ and 0.509 , respectively). Five of 23 (23\%) HNSCC patients with Hp 2-2 or 2-0 had distant metastasis, vs. seven of $23(30 \%)$ HNSCC patients with $\mathrm{Hp} 1-1$ or 2-1 $(\mathrm{p}=0.559)$; eight of $23(35 \%)$ patients with Hp 2-2 or 2-0 died, vs. nine of $23(39 \%)$ patients with Hp 1-1 or 2-1 ( $p=0.76)$. Patients with distant metastasis received palliative treatment. There was a significant difference in terms of risk of locoregional recurrence-free survival between these two groups after adjusting for gender, tumor site, and stage [Hazard ratio (HR) 5.9; 95\% confidence interval (CI) 1.1-6.65; $\mathrm{p}=0.038$; Table 4]. The risk was still higher for the patients with $\mathrm{Hp}$ 2-2 or 2-0 after further adjusting for age and treatment modality (HR 7.6;95\% $\mathrm{Cl}, 1.2-46 ; \mathrm{p}=0.028$ ) with respect to locoregional recurrence-free survival. The HR was not significantly increased in distant metastasis-free survival and overall survival ( $p=0.339$ and 0.776 , respectively; Table 4).

\section{Discussion}

The genotypes of $\mathrm{Hp}$ can be readily determined (12-15) using PCR and electrophoresis. Our data demonstrated that the pattern of Hp genotype distribution in patients with HNSCC was not different from that of healthy individuals. The distribution of Hp genotypes in healthy individuals and patients with HNSCC was in Hardy-Weinberg equilibrium. Although previous studies showed the relationship between specific $\mathrm{Hp}$ genotypes and cancer, this phenomenon was not observed in HNSCC (3).

Our previous study showed that $\mathrm{Hp}$ 2-2 was a negative prognostic factor for nasopharyngeal carcinoma (16). Gast et al. also reported that Hp 2-2 was associated with poor survival in breast cancer patients (17). We divided our HNSCC patients into two groups because of these studies. With matched-pair analysis, 23 patients with Hp 2-2 or 2-0 were pair-matched by gender, primary tumor site, and stage to 23 patients with $\mathrm{Hp}$ 1-1 or 2-1. The patient demographics presented in Table 1 show that the two groups were similar. The two populations could be compared with respect to recurrence, distant metastasis, and overall survival. Figure 3 shows that HNSCC patients with $\mathrm{Hp}$ 2-2 or 2-0 had poor locoregional recurrence survival rates $(p=0.02)$. Using gender, primary tumor site, and overall stage as covariates, $\mathrm{Hp} \mathrm{2-2}$ or 2-0 was an independent prognostic factor for locoregional recurrence ( $H R=5.9 ; p=0.038$ ). The risk was still higher in patients with $\mathrm{Hp}$ 2-2 or 2-0 after further adjusting for age and treatment modality ( $H R$ 7.6; $p=0.028$ ) with respect to locoregional recurrence-free survival. Although there was greater recurrence in HNSCC patients with $\mathrm{Hp} 2-2$ or 2-0, the survival rates were not statistically significant. Seven of $10(70 \%)$ recurrent HNSCC patients with $\mathrm{Hp}$ 2-2 or 2-0 underwent salvage surgery and adjuvant therapy and three patients received salvage chemoradiotherapy. In the Hp 2-2/2-0 group, five HNSCC patients with recurrent tumor were eventually successfully salvaged. This may be one of the reasons why there was no significant difference in survival rates between these two groups.

Some studies investigated the relationship between $\mathrm{Hp}$ concentrations and both HNSCC and nasopharyngeal carcinoma $(10,11)$. Free hemoglobin promotes the accumulation of free radicals and other harmful reactive oxygen species, as well as the breakdown of erythrocytes in interstitial fluid resulting in hemoglobin-mediated free radical formation (18). Plasma Hp is a major antioxidant and can protect against hemoglobin-mediated lipid peroxidation (19). Lim et al. (20) showed that in $\mathrm{Hp}$ knockout mice, more deaths and renal damage occurred during hemolysis. The refer- 

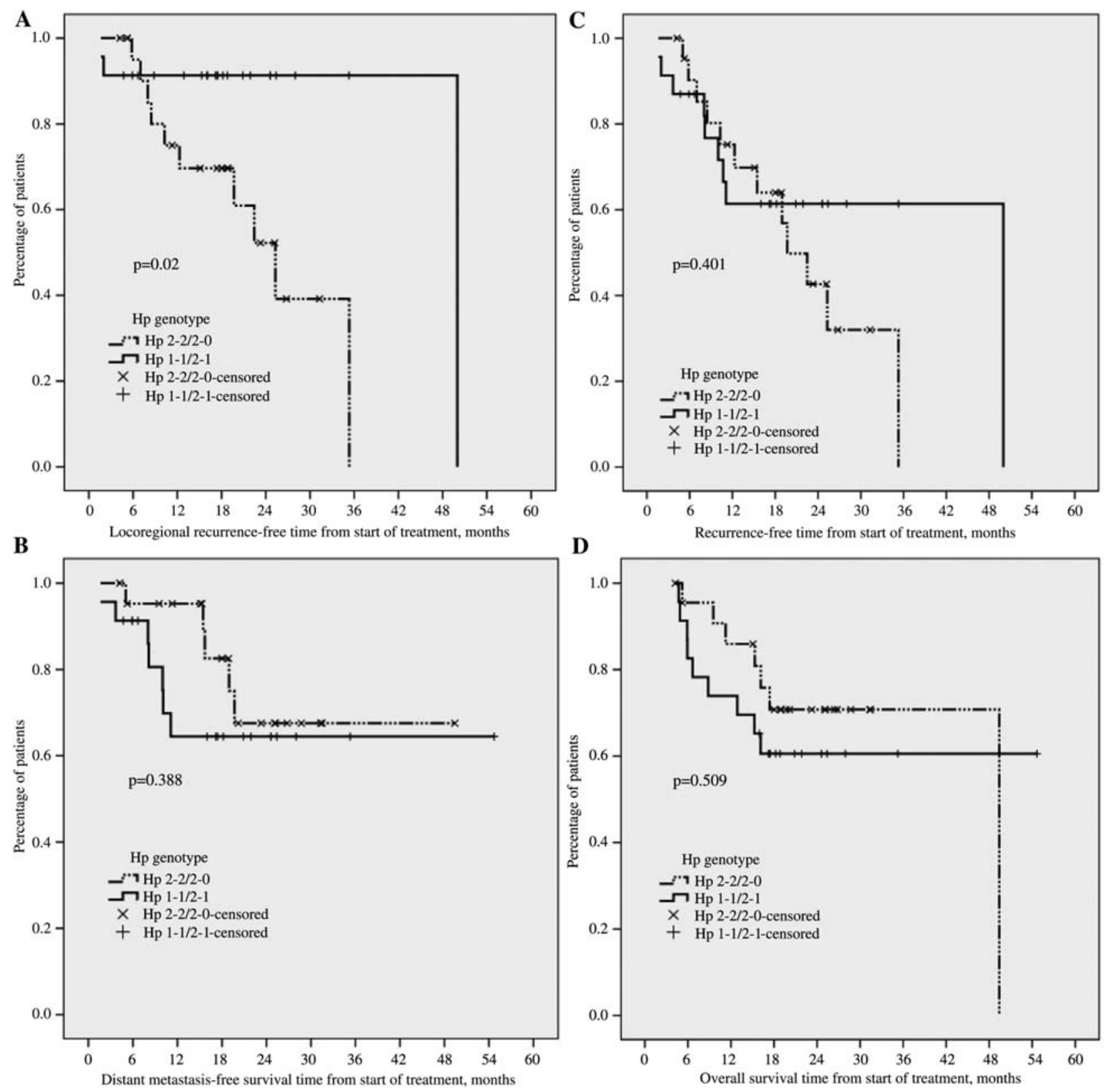

Figure 3 Survival curves of different haptoglobin genotypes.

(A) Locoregional recurrence-free survival; (B) distant metastasis-free survival; (C) recurrence-free survival and (D) overall survival in patients with head and neck squamous cell carcinoma with different haptoglobin genotypes.

ence range for $\mathrm{Hp}$ is lower in individuals with $\mathrm{Hp} 2-2$ and this may result in reduced protection against free radicals $(6,21)$. The antioxidative capacity of body fluids is less efficient in $\mathrm{Hp} \mathrm{2-2}$ individuals. This finding was attributed to the fact that the Hp 2-2 phenotype produces higher molecular mass species (>200 kDa) which restrict their distribution in interstitial fluid (6). Tseng et al. (7) reported that the ranking of the antioxidant activity was as follows: $\mathrm{Hp} 1-1>\mathrm{Hp} 2-1>\mathrm{Hp}$ 2-2 in an vitro study. Also, transfected Chinese ovary cell (CHO) with Hp 1-1 expression had increased tolerance against oxidative stress than $\mathrm{CHO}$ cells expressing other Hp genotypes. Furthermore, individuals with $\mathrm{Hp}$ 2-0 have extremely low concentrations of $\mathrm{Hp}$ (14). Greater locoregional recurrence rates in HNSCC patients with $\mathrm{Hp} \mathrm{2-2}$ or 2-0 may be partially attributed to the poor antioxidant capacity of $\mathrm{Hp} \mathrm{2-2}$ and 2-0 type when compared with other Hp types. Individuals with a $\mathrm{Hp} \mathrm{2-2}$ or 2-0 genotype have lower $\mathrm{Hp}$ concentrations and therefore, less efficient hemoglobin-binding capacity. This results in some degree of heme iron accumulation as shown by the association of Hp 2-2 with higher serum iron, higher transferrin saturation, and higher serum ferritin concentrations $(20,22)$. Iron promotes neoplastic cell growth and accumulates in cancer cells more readily than in normal cells, and excess iron may suppress the tumoricidal activity of macrophages and interfere with lymphocyte trafficking $(23,24)$.

$\mathrm{Hp}$ is involved in arterial restructuring by facilitating cell migration through the accumulation of a temporary gelatin matrix. It has been suggested that $\mathrm{Hp}$ may be involved in other vascular and non-vascular processes such as angiogenesis and tumor cell invasion 
Table 3 Failure patterns of patients with HNSCC according to Hp genotype.

\begin{tabular}{|c|c|c|c|}
\hline & \multicolumn{2}{|l|}{ Genotype } & \multirow[t]{2}{*}{$\mathrm{p}$-Value } \\
\hline & $\begin{array}{l}\text { Hp 1-1, 2-1 } \\
(\mathrm{n}=23)^{\mathrm{a}}\end{array}$ & $\begin{array}{l}\text { Hp 2-2, 2-0 } \\
(\mathrm{n}=23)^{\mathrm{b}}\end{array}$ & \\
\hline \multicolumn{4}{|l|}{ Failure patterns } \\
\hline Locoregional & $3(13)$ & $10(44)$ & 0.022 \\
\hline Distant metastasis & $7(30)$ & $5(23)$ & 0.559 \\
\hline Locoregional and distant metastasis & $1(4)$ & $1(4)$ & 1 \\
\hline \multicolumn{4}{|l|}{ Survival status } \\
\hline Death & $9(39)$ & $7(30)$ & 0.536 \\
\hline
\end{tabular}

Values are given as number (percentage). aFive patients with Hp 1-1 genotype and 18 patients with Hp 2-1; ${ }^{\text {b}} 20$ patients with Hp 2-2 genotype and three patients with Hp 2-0 genotype. HNSCC, head and neck squamous cell carcinoma; Hp, haptoglobin.

Table 4 HR of locoregional recurrence, distant metastasis and overall death from disease associated with Hp genotype 2-2 or 2-0 vs. Hp genotype 1-1 or 2-1 in head and neck cancer.

\begin{tabular}{|c|c|c|c|c|c|c|}
\hline Analysis & $\begin{array}{l}\text { Locoregional recurrence } \\
\text { HR }(95 \% \mathrm{Cl})\end{array}$ & p-Value & $\begin{array}{l}\text { Distant metastasis } \\
\text { HR }(95 \% \mathrm{Cl})\end{array}$ & p-Value & $\begin{array}{l}\text { Overall death } \\
\text { HR }(95 \% \mathrm{Cl})\end{array}$ & $\mathrm{p}$-Value \\
\hline $\begin{array}{l}\text { Cox regression analysis on } \\
\text { matching variables } \\
\text { (gender, disease site, stage) }\end{array}$ & $5.9(1.104-6.65)$ & 0.038 & $0.4(0.121-1.465)$ & 0.174 & $0.8(0.296-2.385)$ & 0.743 \\
\hline $\begin{array}{l}\text { Adjusted for age and treatment } \\
\text { modality }\end{array}$ & $7.6(1.2-46)$ & 0.028 & $0.55(0.157-1.894)$ & 0.339 & $1.17(0.416-3.285)$ & 0.776 \\
\hline
\end{tabular}

$\mathrm{HR}$, hazard ratio; $\mathrm{Hp}$, haptoglobin; $95 \% \mathrm{Cl}, 95 \%$ confidence interval.

(8). Hp 2-2 stimulates endothelial cell differentiation and has more angiogenic effects than other Hp phenotypes, both in in-vitro and in-vivo models of angiogenesis (9). Kuhajda et al. (25) showed that decreased tumor expression of Hp-related protein is associated with better recurrence-free survival in patients with breast cancer. The expression of a peak at mass-tocharge ratio $9198>20$, identified as a $\mathrm{Hp} \alpha-1$ chain, has better recurrence free survival, and 63 breast cancer patients with $\mathrm{Hp} 2-2$, which lacked an $\mathrm{Hp} \alpha-1$ chain, conferred a poor prognosis (17). Collectively, these observations may present a possible mechanism for free radical generation and cancer progression in patients with $\mathrm{Hp}$ 2-2.

There are several limitations of this study. First, the oxidative stress markers and serum $\mathrm{Hp}$ concentration were not measured. Second, the number of cases was limited. We tried to limit the effect of potential confounders by matching analysis. The matching for three variables known to affect prognosis (gender, tumor site, and overall stage) renders it more likely to detect any differences due to $\mathrm{Hp}$ polymorphism.

Patients with $\mathrm{Hp} \mathrm{2-2}$ or 2-0 have more locoregional recurrences and significantly increased HRs in multivariate analysis. The Hp genotype may be a prognostic factor in HNSCC.

\section{Conflict of interest}

The authors have no relevant financial interest in this article.

\section{Acknowledgements}

This study was supported by grant No. DTCRD 94 (2)-11 from the Buddhist Tzu Chi Dalin General Hospital.

\section{References}

1. Cancer Registry Annual Report, Republic of China, 2001. Department of Health, The Executive Yuan, 2004.

2. Marinkovic S, Baumann H. Structure, hormonal regulation, and identification of the interleukin-6- and dexamethasone-responsive element of the rat haptoglobin gene. Mol Cell Biol 1990;10:1573-83.

3. Carter K, Worwood M. Haptoglobin: a review of the major allele frequencies worldwide and their association with diseases. Int J Lab Hematol 2007;29:92-110.

4. Delanghe JR, Langlois MR, Boelaert JR, Van Acker J, Van Wanzeele F, van der Groen G, et al. Haptoglobin polymorphism, iron metabolism and mortality in HIV infection. AIDS 1998;12:1027-32.

5. Van Vlierberghe H, Langlois M, Delanghe J. Haptoglobin polymorphisms and iron homeostasis in health and in disease. Clin Chim Acta 2004;345:35-42.

6. Langlois MR, Delanghe JR. Biological and clinical significance of haptoglobin polymorphism in humans. Clin Chem 1996;42:1589-600.

7. Tseng CF, Lin CC, Huang HY, Lin HC, Mao SJ. Antioxidant role of human haptoglobin. Proteomics 2004;4: 2221-8.

8. Cid MC, Grant DS, Hoffman GS, Auerbach R, Fauci AS, Kleinman HK. Identification of haptoglobin as an angiogenic factor in sera from patients with systemic vasculitis. J Clin Invest 1993;91:977-85.

9. de Kleijn DPV, Smeets MB, Kemmeren PP, Lim SK, Van Middelaar BJ, Velema E, et al. Acute-phase protein haptoglobin is a cell migration factor involved in arterial restructuring. FASEB J 2002;16:1123-5.

10. Wolf GT, Wolfe RA. Circulating immune-complexes in patients with nasopharyngeal carcinoma. Laryngoscope 1990;100:302-8.

11. Gallo O, Gori AM, Attanasio M, Martini F, Giusti B, Brunelli $T$, et al. Interleukin- 6 and acute-phase proteins in head and neck cancer. Eur Arch Otorhinolaryngol 1995; 252:159-62.

12. Koch W, Latz W, Eichinger M, Roguin A, Levy AP, Schomig $A$, et al. Genotyping of the common haptoglobin Hp 1/2 polymorphism based on PCR. Clin Chem 2002; 48:1377-82. 
13. Koch W, Latz W, Eichinger M, Gschwender C, Teige B, Schoming $A$, et al. Haptoglobin gene subtyping by restriction enzyme analysis. Clin Chem 2003;49:1937-40.

14. Koda Y, Soejima M, Yoshioka N, Kimura H. The haptoglobin-gene deletion responsible for anhaptoglobinemia. Am J Hum Genet 1998;62:245-52.

15. Koda Y, Watanabe $Y$, Soejima M, Shimada E, Nishumura M, Morishita K, et al. Simple PCR detection of haptoglobin gene deletion in anhaptoglobinemic patients with antihaptoglobin antibody that causes anaphylactic transfusion reactions. Blood 2000;95:1138-43.

16. Lee CC, Lin HY, Hung SK, Li DK, Ho HC, Lee MS, et al. Haptoglobin genotypes in nasopharyngeal carcinoma. Int J Biol Marker 2009;24:32-7.

17. Gast MC, van Tinteren $H$, Bontenbal M, van Hoesel RQ, Nooij MA, Rodenhuis S, et al. Haptoglobin phenotype is not a predictor of recurrence free survival in high-risk primary breast cancer patients. BMC Cancer 2008;8:389.

18. Sadrzadeh SM, Graf E, Panter SS, Hallaway PE, Eaton JW. Hemoglobin. A biologic fenton reagent. J Biol Chem 1984;259:14354-6.
19. Gutteridge JM. The antioxidant activity of haptoglobin towards haemoglobin-stimulated lipid peroxidation. Biochim Biophys Acta 1987;917:219-23.

20. Lim YK, Jenner A, Ali AB, Wang T, Hsu SI, Chong SM, et al. Haptoglobin reduces renal oxidative DNA and tissue damage during phenylhydrazine-induced hemolysis. Kidney Int 2000;58:1033-44.

21. Thomas L. Haptoglobin/Hämopexin. In: Thomas L, editor. Labor und Diagnose, 4th ed. Marburg: Medizinische Verlagsgesellschaft 1992:813-20.

22. Na N, Delanghe JR, Taes YE, Torck M, Baeyens WR, Ouyang J. Serum vitamin C concentration is influenced by haptoglobin polymorphism and iron status in Chinese. Clin Chim Acta 2003;365:319-24.

23. Weinberg ED. The role of iron in cancer. Eur $\mathrm{J}$ Cancer Prev 1996;5:19-36.

24. Toyokuni S. Iron-induced carcinogenesis: the role of redox regulation. Free Rad Biol Med 1996;20:553-66.

25. Kuhajda FP, Katumuluwa Al, Pasternack GR. Expression of haptoglobin-related protein and its potential role as a tumor antigen. Proc Natl Acad Sci USA 1989;86:1188-92. 